\title{
Gadolinium and nephrogenic systemic fibrosis: The evidence of things not seen
}

NSF: A

devastating

new disease

in patients

with renal

dysfunction

and exposure

to gadolinium
Now faith is the substance of things hoped for, the evidence of things not seen.

HEBREWS 11:1.

INCE THE FIRST CASE appeared in 1997,1 $\checkmark$ nephrogenic systemic fibrosis (NSF) has been detected with increasing frequency in patients with chronic kidney disease. Recognition that this condition affects more than just the skin led to the change in its name from "nephrogenic fibrosing dermopathy" to "nephrogenic systemic fibrosis."

In this issue, Issa and colleagues ${ }^{2}$ review this devastating new disease and discuss its association with gadolinium exposure.

See related article, page 95

\section{NSF RESEMBLES OTHER FIBROSING DISORDERS}

The clinical presentation of NSF most closely resembles that of scleromyxedema or scleroderma. 1 However, the face is spared in patients with NSF except for yellow plaques on the sclerae, a frequent finding. Monoclonal gammopathy (which may be associated with scleromyxedema) and Raynaud's phenomenon (which often is associated with scleroderma) usually are absent in NSF. ${ }^{3}$

A set of histologic findings differentiates NSF from other fibrosing disorders. Skin biopsy reveals fibrosis and elastosis, often with mucin deposition. If NSF is suspected, immunohistochemical stains for CD34, $\mathrm{CD} 45 \mathrm{RO}$, and type I procollagen should be performed to look for dermal spindle cells (presumably "circulating fibrocytes") coexpressing these markers. Histiocytic cells and dermal dendrocytes expressing CD68 and factor XIIIa have also been described in NSF skin lesions, but other inflammatory cells usually are absent. 4 However, the histologic changes of NSF are difficult to distinguish from those of scleromyxedema. ${ }^{5}$

Thus, as with scleroderma, the diagnosis of NSF remains clinical. Skin biopsy, even of an affected area, occasionally may yield nondiagnostic findings. Histologic findings serve to confirm the diagnosis of NSF in the appropriate clinical setting.

\section{RISK FACTORS FOR NSF: POSSIBLE ASCERTAINMENT BIAS}

\section{Renal dysfunction}

Because cases of NSF have been searched for only in patients with chronic kidney disease, reported cases have been found only in this patient population. A major limitation of most published case series is that cases have been gathered from among those with histologic confirmation of NSF, and "controls" have been gathered from the remainder of the population receiving dialysis treatment without confirmation by physical examination of the absence of cutaneous changes of NSF.

Most cases have been found in those with stage 5 chronic kidney disease (creatinine clearance $<15 \mathrm{~mL} / \mathrm{min}$ or requiring dialysis). However, cases have been described in patients with stage 4 chronic kidney disease 
(creatinine clearance $15-29 \mathrm{~mL} / \mathrm{min}$ ) and, occasionally, in those with lesser degrees of impaired renal function.

Despite the ascertainment bias in identifying cases, this greater prevalence of NSF with lesser renal function suggests a role for renal dysfunction in the pathogenesis of NSF.

\section{Gadolinium exposure}

To date, nearly all patients who have developed NSF have had known exposure to gadolinium-containing contrast agents. Gadolinium has been found in tissue of patients with NSF,6,7 yielding the postulate that gadolinium drives tissue fibrosis.

More patients with chronic kidney disease who developed NSF had been exposed to gadodiamide (Omniscan) than to other gadoliniumcontaining contrast agents, leading to the hypothesis that less-stable gadolinium-chelate complexes release greater amounts of free gadolinium, which then deposits in tissue and triggers fibrosis. However, it has not yet been determined that the gadolinium deposited in tissue is in the free form and not bound to chelate. Furthermore, this attractive hypothesis must be tempered by the recognition that NSF also has developed after exposure to gadopentetate dimeglumine (Magnevist), a more stable gadolinium-chelate complex than gadodiamide. 8 The greater number of patients who have developed NSF after gadodiamide exposure may reflect the relative use of these contrast agents in radiology practice.

It is important to be aware that gadolinium-containing contrast agents are used in more than just magnetic resonance imaging (MRI) and magnetic resonance angiography (MRA). Because gadolinium also blocks transmission of $\mathrm{x}$-rays, radiologists occasionally have used gadolinium-containing contrast agents for angiography, venography, fistulography, and computed tomography in patients for whom use of iodinated contrast agents is contraindicated. Thus, a patient with chronic kidney disease may have received a gadoliniumcontaining contrast agent even if no magnetic resonance study had been performed.

Assessment of tissue gadolinium content may confirm prior exposure to a gadoliniumcontaining contrast agent if the patient does not recall having undergone an imaging study.
In the one report that claims the development of NSF in two patients without prior gadolinium exposure, tissue was not assessed for gadolinium content. ${ }^{9}$

No study has yet been performed to assess the relative prevalence of NSF among patients with different stages of chronic kidney disease who have been exposed to gadolinium-containing contrast agents. Thus, it is impossible to ascertain a threshold of renal dysfunction above which the use of gadolinium-containing contrast agents might be safe.

In 90 patients with stage 5 chronic kidney disease, we found that $30 \%$ of those who previously had undergone gadolinium-enhanced imaging studies developed cutaneous changes of NSF; the relative risk of developing these skin changes after exposure to a gadoliniumcontaining contrast agent was 10.7 (95\% confidence interval 1.5-6.9).8

Thus, it is essential that guidelines for the use of these contrast agents be formulated and implemented. Caution must be observed when administering a gadolinium-containing contrast agent to a patient with any degree of renal dysfunction. These patients must be informed of the possible risk of developing NSF, and appropriate follow-up must be conducted to assess for potential changes of NSF.

\section{Other possible risk factors}

Not all patients with chronic kidney disease who are exposed to gadolinium-containing contrast agents develop NSF: factors other than the degree of renal dysfunction must be involved in the pathogenesis of this condition.

Exposure to medications commonly taken by patients with chronic kidney disease, such as erythropoietin 10 and iron supplements, 11 has been suggested as a contributing factor. However, these medications are so widely used that this exposure is unlikely to explain why some patients develop NSF after receiving gadolinium-containing contrast agents and others do not.

Interestingly, lanthanum carbonate (Fosrenol) was approved by the US Food and Drug Administration in 2004 for use as a phosphate binder in patients with stage 5 chronic kidney disease. Since lanthanum and gadolinium both are rare earth metals of the lanthanide series, one might speculate that 
lanthanum deposition in tissue could produce similar changes or could potentiate those induced by gadolinium.

Future prospective case-control studies need to address risk factors for the development of NSF.

\section{EFFECTIVE TREATMENT NEEDED}

Because NSF imposes a markedly increased rate of death and devastating morbidity, ${ }^{8}$ efforts must be directed toward preventing its development and treating those who already are affected. So far, no treatment has been universally effective in reversing the fibrotic changes of NSF. Potentially effective therapeutic agents must be identified and studied in these patients.

Although performing hemodialysis promptly after the use of a gadolinium-containing contrast agent would appear to be a prudent clinical practice, there are no data to suggest that it is effective in preventing NSF. If free gadolinium disassociates from its chelate and deposits rapidly in tissue, it is

\section{REFERENCES}

1. Cowper SE, Robin HS, Steinberg SM, Su LD, Gupta S, LeBoit PE. Scleromyxoedema-like cutaneous diseases in renal-dialysis patients. Lancet 2000; 356:1000-1001.

2. Issa N, Poggio E, Fatica R, Patel R, Ruggieri PM, Heyka RJ. Nephrogenic systemic fibrosis and its association with gadolinium exposure during MRI. Cleve Clin J Med 2008; 75:95-111.

3. Moschella SL, Kay J, Mackool BT, Liu V. Case records of the Massachusetts General Hospital. Weekly clinicopathological exercises. Case 35-2004. A 68-year-old man with end-stage renal disease and thickening of the skin. $\mathrm{N} \mathrm{Engl}$ J Med 2004; 351:2219-2227.

4. Cowper SE, Su LD, Bhawan J, Robin HS, LeBoit PE. Nephrogenic fibrosing dermopathy. Am J Dermatopathol 2001; 23:383-393.

5. Kucher C, Xu X, Pasha T, Elenitsas R. Histopathologic comparison of nephrogenic fibrosing dermopathy and scleromyxedema. J Cutan Pathol 2005; 32:484-490.

6. High WA, Ayers RA, Chandler J, Zito G, Cowper SE. Gadolinium is detectable within the tissue of patients with nephrogenic systemic fibrosis. J Am Acad Dermatol 2007; 56:21-26. unclear that hemodialysis could be performed soon enough to prevent this deposition. Furthermore, hemodialysis is not without associated potential risks and morbidity, especially in people with chronic kidney disease who are not already receiving hemodialysis. Thus, at present, avoiding the use of gadolinium-containing contrast agents in patients with chronic kidney disease appears to be the best preventive strategy.

\section{A NAME CHANGE}

Over the past decade, much has been learned about the clinical manifestations, course, and pathogenesis of NSF. However, the term "nephrogenic" in the name of this disease is misleading, in that this fibrosing disorder is not caused by the kidneys. Although some degree of renal dysfunction appears to be necessary for NSF to develop, the presence of gadolinium in tissue seems to drive fibrosis. Thus, it is time that "nephrogenic systemic fibrosis" be renamed more precisely as "gadolinium-associated systemic fibrosis" or "GASF."

7. Boyd AS, Zic JA, Abraham JL. Gadolinium deposition in nephrogenic fibrosing dermopathy. J Am Acad Dermatol 2007; 56:27-30.

8. Todd DJ, Kagan A, Chibnik LB, Kay J. Cutaneous changes of nephrogenic systemic fibrosis: predictor of early mortality and association with gadolinium exposure. Arthritis Rheum 2007; 56:3433-3441.

9. Wahba IM, Simpson EL, White K. Gadolinium is not the only trigger for nephrogenic systemic fibrosis: insights from two cases and review of the recent literature. Am J Transplant 2007; 7:2425-2432.

10. Swaminathan S, Ahmed I, McCarthy JT, et al. Nephrogenic fibrosing dermopathy and high-dose erythropoietin therapy. Ann Intern Med 2006; 145:234-235.

11. Swaminathan S, Horn TD, Pellowski D, et al. Nephrogenic systemic fibrosis, gadolinium, and iron mobilization. $\mathrm{N}$ Engl J Med 2007; 357:720-722.

ADDRESS: Jonathan Kay, MD, Rheumatology Unit, Massachusetts General Hospital, 55 Fruit Street, Yawkey 2-174, Boston, MA 02114; e-mail jkay@partners.org.
Use caution when giving a gadolinium contrast agent to a patient who has any degree of renal dysfunction 